ity and $t$ independence of the operator $P(x, D)$. This permits $P$ to be a quite general operator. Finally, if $P_{1}$ and $P_{2}$ reduce to initial value problems, a similar argument is applicable. In this case, conditions on the known function $u(x, t)$ (or $v(x, t)$ ) can be imposed on the unknown function $v(x, t)$ (or $u(x, t)$ ). With no boundary conditions on $u$ and $v$, it is no longer necessary to require that $\phi(x)$ satisfy a boundary condition.

OAKLAND UNIVERSITY

\title{
LOCALLY NICE EMBEDDINGS IN CODIMENSION THREE ${ }^{1}$
}

\author{
BY J. L. BRYANT AND C. L. SEEBECK, III
}

Communicated by O. G. Harrold, October 16, 1967

1. Introduction. Suppose that $K$ is a $k$-dimensional compactum in the interior of a topological $q$-manifold $Q, q-k \geqq 3$. Following Hempel and McMillan [3], we say that $K$ is locally nice in $Q$ if $Q-K$ is 1-ULC. Similarly, an embedding $f: K \rightarrow \operatorname{Int} Q$ is said to be locally nice if $Q-f(K)$ is 1 -ULC.

In [1] the authors showed that a locally nice embedding of a compact $k$-dimensional polyhedron $K$ into Int $Q$, where $Q$ is a PL $q$-manifold, is $\epsilon$-tame whenever $q \geqq 5$ and $2 k+2 \leqq q$. In this announcement we outline the proof that the same is true for embeddings in codimension at least three if $K$ is a compact PL manifold. Specifically, our main result is

Theorem 1. Suppose that $M$ and $Q$ are $P L$ manifolds of dimensions $m$ and $q$, respectively, with $M$ compact, $q \geqq 5$, and $q-m \geqq 3$, and $f: M \rightarrow I n t Q$ is a locally nice embedding. Then $f$ is $\epsilon$-tame.

The following two corollaries serve to demonstrate the usefulness of Theorem 1 as applied to some special locally nice embeddings.

Corollary 1.1. Suppose that $P$ is a locally tame $(q-1)$-complex in the $P L q$-manifold $Q, q \geqq 5$, and $M$ is a compact $P L$ m-manifold in Int $Q, q-m \geqq 3$, such that $M-P$ is locally tame. Then $M$ is $\epsilon$-tame.

${ }_{1}$ This research was supported in part by NSF grant GP-5458. 
For example, let $Q=E^{q}, P=E^{q-1}$, and $M \subset P$.

Corollary 1.2. Suppose $M^{m} \subset E^{q}$ and $N^{n} \subset E^{r}$ with $m \leqq q-1$, $n \leqq r-2$. Then $M \times N \subset E^{q} \times E^{r}$ is $\epsilon$-tame.

Proof. If $q+r \leqq 4$, the result is standard. McMillan has shown [7] that in any case, $M \times N$ is locally nice in $E^{q} \times E^{r}$.

Definitions. $M$ and $Q$ will denote PL manifolds of dimensions $m$ and $q$, respectively, with or without boundary and $M$ compact, and $I$ will denote the unit interval $[0,1]$. If $X \subset Q$ with $\mathrm{Cl}(X)$ compact and $\epsilon>0$, then an $\epsilon$-push of the pair $(Q, X)[2]$ is a homeomorphism of $Q$ onto itself that is $\epsilon$-isotopic to the identity by an isotopy $h_{t}(t \in I)$ of $Q$ such that each $h_{t}$ is the identity outside $N_{\epsilon}(X)$, the $\epsilon$-neighborhood of $X$. An embedding $f: M \rightarrow Q$ is said to be $\epsilon$-tame if for each $\epsilon>0$ there exists an $\epsilon$-push $h$ of $(Q, f(M))$ such that $h f: M \rightarrow Q$ is PL.

The proof of Theorem 1 depends upon the following two theorems, the first of which was proved in [1].

ThEOREM 2. Suppose that $A$ is a compact k-dimensional $A N R$ in Int $Q$ with $q \geqq 5$ and $q-k \geqq 3$ such that $Q-A$ is $1-U L C$. For each $\epsilon>0$, there exists $\delta>0$ such that if $f: A \rightarrow \operatorname{Int} Q$ is a $\delta$-homeomorphism and $U$ is an open subset of $Q$ containing $f(A)$, then there is an $\epsilon$-push $h$ of $(Q, A)$ such that $h(U) \supset A$.

THEOREM 3. Suppose that $f: M \rightarrow Q$ is an embedding with $q-m \geqq 3$. For each $\epsilon>0$ there exists $\delta>0$ such that if $g_{0}, g_{1}: M \rightarrow$ Int $Q$ are $P L$ embeddings with $d\left(g_{i}(x), f(x)\right)<\delta, i=0,1$, for each $x \in M$, then there exists an $\epsilon$-push $h$ of $(Q, f(M))$ such that $h g_{0}=g_{1}$.

Theorem 3 is proved by first using Homma's techniques [5] to find a $\delta>0$ corresponding to $\epsilon>0$ such that if $g_{0}, g_{1}: M \rightarrow \operatorname{Int} Q$ are PL embeddings within $\delta$ of $f$, then there exist $m+1$ " $\epsilon /(m+1)$-concordances" of $M$ in Int $Q$ starting with $g_{0}$ and ending with $g_{1}$. A result of Hudson's [6] then allows us to replace these concordances by $\epsilon /(m+1)$-ambient isotopies of $Q$ that compose to give the desired $\epsilon$-push. Theorems 2 and 3 are now combined to prove a

Lemma. Suppose that $f: M \rightarrow \operatorname{Int} Q, q \geqq 5$ and $q-m \geqq 3$, is a locally nice embedding. For each $\epsilon>0$ and $\eta>0$, there exists $\delta=\delta(f, \epsilon)>0$ such that if $g: M \rightarrow$ Int $Q$ is a $P L$ embedding within $\delta$ of $f$, then there exists an $\epsilon$-push $h$ of $(Q, f(M))$ such that $d(h f(x), g(x))<\eta$ for each $x \in M$.

We proceed now much as Homma did in [4]. Given a locally nice embedding $f: M \rightarrow \operatorname{Int} Q$ and $\epsilon>0$, we let $\delta=\delta(f, \epsilon / 4)>0$ be chosen as in the Lemma and $g: M \rightarrow$ Int $Q$ be a PL embedding within $\delta$ of $f$. 
Theorem 3 and the Lemma give precisely the amount of uniformity required to obtain two sequences of pushes $\phi^{1}, \phi^{2}, \cdots$ and $\psi^{1}$, $\psi^{2}, \cdots$ of $(Q, f(M)$ (converging to $\epsilon / 2$-pushes $\phi$ and $\psi$ of $(Q, f(M))$, respectively, such that $\phi f=\psi g$. The desired $\epsilon$-push of $(Q, f(M))$ is the composition $h=\psi^{-1} \phi$.

Complete proofs of these results will appear in full elsewhere.

Added in proof. Since this announcement was submitted, some errors in the proof of Homma's approximation theorem have been discovered. Should these discrepancies not be rectified, then the restriction $q-m \geqq 3$ must be replaced by $m \leqq(2 / 3) q-1$ in the statements of Theorem 1 and the corollaries.

\section{REFERENCES}

1. J. L. Bryant and C. L. Seebeck, III, Locally nice embeddings of polyhedra, Quart. J. Math. Oxford Ser., (to appear).

2. H. Gluck, Embeddings in the trivial range, Ann. of Math. (2) 81 (1965), 195210.

3. J. P. Hempel and D. R. McMillan, Jr., Locally nice embeddings of manifolds, Amer. J. Math. 88 (1966), 1-19.

4. T. Homma, On the imbedding of polyhedra in manifolds, Yokohama Math. J. 10 (1962), 5-10.

5. - Piecewise linear approximations of embeddings of manifolds, Mineographed notes, Florida State University, 1965.

6. J. F. P. Hudson, Concordance and isotopy of PL embeddings, Bull. Amer. Math. Soc. 72 (1966), 534-535.

7. D. R. McMillan, Jr., A criterion for cellularity in a manifold, Ann. of Math. (2) 79 (1964), 327-337.

Florida State University, and

Michigan State University

\section{ERRATUM, VOLUME 73}

Index to Volume 73, p. 1004.

Line 29 should read:

Kahn, Peter J. Chern numbers and oriented homotopy type, 932 